\title{
Minimizing the Surface Roughness for Silicon Ablation with Ultrashort Laser Pulses
}

\author{
Matthias Domke* ${ }^{* 1}$ Giovanni Piredda ${ }^{* 1}$, Johann Zehetner ${ }^{* 1}$, Sandra Stroj ${ }^{* 1}$ \\ ${ }^{*}$ J Josef Ressel Center for material processing with ultrashort pulsed lasers, University of Applied \\ Sciences Vorarlberg, 6850 Dornbirn, Austria \\ E-mail: matthias.domke@fhv.at
}

\begin{abstract}
The application of ultrashort pulsed lasers for surface patterning enables precise control of the ablation depth. The goal of this study is to find the optimal combination of pulse-to-pulse distance and fluence in order to minimize the surface roughness for Si ablation. A Si wafer with a thickness of $525 \mu \mathrm{m}$ was irradiated using an ultrafast laser with a pulse duration of $380 \mathrm{fs}$ at a wavelength of $520 \mathrm{~nm}$ and a pulse frequency of $200 \mathrm{kHz}$. An area of $50 \times 50 \mu \mathrm{m}^{2}$ was ablated to a depth of about $10 \mu \mathrm{m}$ using different pulse and line distances $d_{p, l}$ and peak fluences $F_{0}$. Confocal microscopy was used to investigate the surface profiles and to determine the surface roughness. For a fixed fluence, two pulse distances can be found where the formation of furrows can be suppressed. The global surface roughness minimum of $220 \mathrm{~nm}$ was achieved using a pulse and line distance of $d_{p, l} \approx 0.67 w_{0}$ and a fluence of $F_{0} \approx 2.8 \mathrm{~J} / \mathrm{cm}^{2}$. The influence of the furrows on the surface roughness is negligible. For fluences above $8 \mathrm{~J} / \mathrm{cm}^{2}$, the surface roughness as a function of the pulse distance has two minima. The results also show that locations of these minima increase with fluence.
\end{abstract}

DOI: $10.2961 /$ jlmn.2016.01.0019

Keywords: ultrashort, pulse, laser, ablation, silicon, surface, structuring, roughness

\section{Introduction}

The application of ultrashort pulsed lasers for surface patterning enables precise control of the ablation depth. A general challenge for high-quality laser ablation is to find the optimal combination of pulse-to-pulse distance and fluence in order to minimize the surface roughness and to maximize throughput as shown e.g. in [1].

In recent years, a simple model to calculate the crater shape has established. With help of this model it was shown that the peak fluence of a Gaussian Beam $F_{0}$ should be $\mathrm{e}^{2}$-times the ablation threshold fluence $F_{t h}$ to achieve the maximum ablation efficiency [1].

In order to minimize the surface roughness, Neuenschwander et al. [1] suggested that the optimum ratio between pulse distance $d_{p}$ and spot radius $w_{0}$ should be $d_{p} / w_{0}>1.0$, and the line distance $d_{l}$ should be $d_{l} / w_{0} \approx 0.5$.

The surface roughness is also influenced by the formation of random or almost periodic holes with a depth of several $\mu \mathrm{m}[2,3]$. Periodic holes can be produced in silicon using ns-lasers [4] and fs-lasers [5]. Sharp conical spikes can be created under $\mathrm{SF}_{6}$ atmosphere [6]. Random formation of holes on ablated surfaces was observed in steel $[7,2]$ and can be found also in silicon [3]. The formation of such laser-induced periodic surface structures (LIPSS) can be used to functionalize surfaces $[4,6,8]$. However, for high quality surface ablation the formation of periodic structures and random holes should be avoided.

The formation of random and periodic holes during surface ablation of silicon was investigated in [3]. Periodic holes with a depth of several $\mu \mathrm{m}$ were observed at a fluence of $1.4 \mathrm{~J} / \mathrm{cm}^{2}$. LIPSS are oriented parallel to the polarization, while periodic holes appear in the furrows. At a fluence of $4.3 \mathrm{~J} / \mathrm{cm}^{2}$, LIPSS can be suppressed and the hole formation is reduced, if the scan direction is oriented perpendicular to the polarization. The surface roughness reduces by a factor of 2 . At $14.1 \mathrm{~J} / \mathrm{cm}^{2}$, the formation of LIPPS can be suppressed in both scan directions, but the surface roughness increases again due to the increasing amount and size of melt ejections.

In [3], the surface roughness was investigated only as a function of fluence at a fixed pulse-to-pulse distance. The goal of this study is to find the optimal combination of pulse-to-pulse distance and fluence for the ablation of silicon in order to minimize the surface roughness.

\section{Material \& Methods}

\subsection{Laser Processing System}

The laser processing machine (3D-Micromac, microSTRUCT vario) has an ultrafast laser source (Spectra Physics, Spirit) with a pulse duration of about $380 \mathrm{fs}$. The laser is operated at a wavelength of $520 \mathrm{~nm}$ and at a pulse frequency of $f_{\text {rep }}=200 \mathrm{kHz}$ in all experiments. The maximum power is $2.0 \mathrm{~W}$. Thus, the maximum pulse energy is $E=10 \mu \mathrm{J}$. The laser beam is scanned across the sample by a galvanometer scanner. The focusing optic is an f-theta lens with a focal length of $100 \mathrm{~mm}$. The focus radius was determined to be $w_{0}=6 \mu \mathrm{m}$ using the method of Liu [9]. All fluence values correspond to the peak fluence $F_{0}$ of the Gaussian beam profile. The laser beam was linearly polarized in all experiments. 


\subsection{Ablation strategy and determination of surface roughness}

The samples used are monocrystalline Si wafers with a thickness of $525 \mu \mathrm{m}$.

An area of $50 \times 50 \mu \mathrm{m}^{2}$ was scanned several times with unidirectional parallel lines. The distance between pulses $d_{p}$ and lines $d_{l}$ was kept equal in all experiments. It should be noted that the laser and the scanner are not synchronized. The start of each line thus varies within the pulse distance (See Fig. 1). The pulses hit the lines on different positions, if the area is scanned several times. The lines remain at the same position.

It was already demonstrated that the orientation of the polarization with respect to the scan direction influences the formation of LIPSS on the ablated surface. LIPPS are rather suppressed if the polarization is oriented perpendicular to the scan direction [3]. This orientation of the polarization was chosen in all experiments (see Fig. 1).

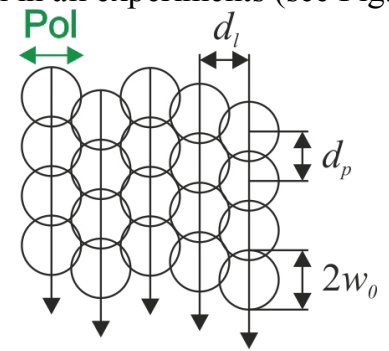

Fig. 1 Ablation strategy. The polarization (green arrow) is oriented perpendicular to the scan direction (black arrows). The distance between pulses $d_{p}$ and lines $d_{l}$ was kept equal in all experiments.

In a first experiment, the pulse distance was fixed to $5 \mu \mathrm{m}$ while the fluence and the number of scans was varied. The depth of the ablated pockets was measured with an optical microscope. The depth was plotted as a function of the number of scans for each fluence. A linear equation was fitted to the data points. This equation was then used to calculate the number of scans to ablate to a depth of $10 \mu \mathrm{m}$ using different fluences and pulse distances.

Then, a second matrix of pockets was ablated, and the surface roughness was measured at the bottom of each ablated surface using confocal microscopy. An example is shown in Fig. 2.

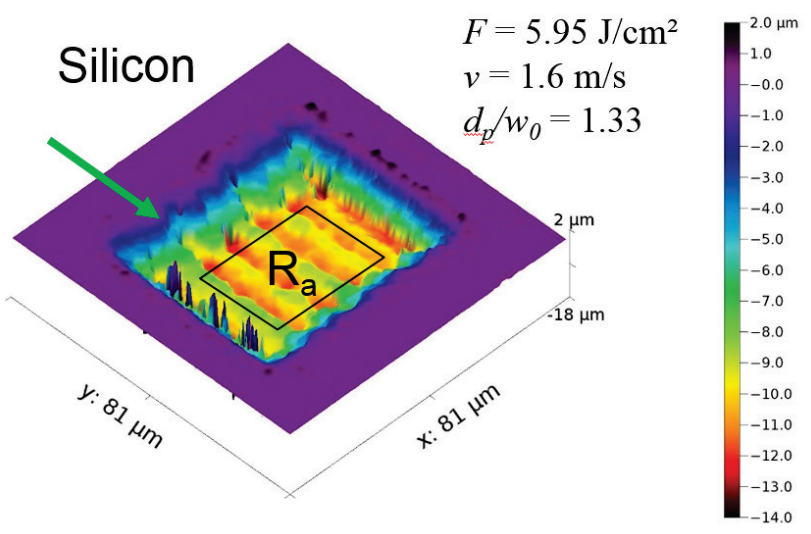

Fig. 2 Confocal microsopy image of an ablated pocket in silicon. The surface roughness $R_{a}$ was determined at the bottom of the ablated surface (black square). The green arrow indicates the scan direction.

\section{Results and discussion}

\subsection{Specific ablation rate of silicon}

Fig. 3 shows the specific ablation rate for silicon measured in $\mathrm{mm}^{3} /(\min \mathrm{W})$ as a function of the fluence. The specific ablation rate increases steeply with the fluence until it reaches its maximum at $2 \mathrm{~J} / \mathrm{cm}^{2}$. The specific ablation rate then remains approximately constant.

According to the model in [1], the specific ablation rate should drop approximately to the half maximum at a fluence of about $14 \mathrm{~J} / \mathrm{cm}^{2}$. The specific ablation rate remains however almost constant above $2 \mathrm{~J} / \mathrm{cm}^{2}$. This could be explained as follows. In the model in [1], it is assumed that the energy penetration depth is constant. Experimental results in [10] show however that the ablation depth per pulse increases nonlinearly with fluence. The energy penetration depth hence cannot be constant for silicon. The results in Fig. 3 indicate that the energy penetration depth seems to increase with the fluence in a way that the specific ablation rate remains almost constant at fluences above $2 \mathrm{~J} / \mathrm{cm}^{2}$.

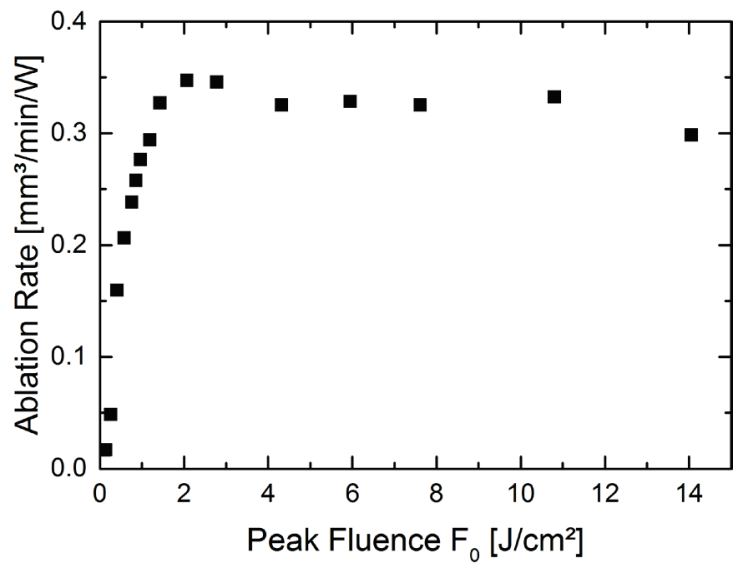

Fig. 3 Specific ablation rate of silicon as function of fluence at a fixed pulse distance of $5 \mu \mathrm{m}$.

\subsection{Investigation of the ablated surface profile}

Fig. 4 shows a matrix of confocal microscopy images of ablated surfaces in silicon, which were irradiated with different fluences and different ratios between pulse distance $d_{p}$ and spot radius $w_{0}$.

At a fluence of $2.8 \mathrm{~J} / \mathrm{cm}^{2}$ (left column), the surface of the ablated pocket appears homogeneous at $d_{p} / w_{0}=0.50$ and $d_{p} / w_{0}=0.67$. Furrows and ridges can be observed at $d_{p} / w_{0}=0.83$. Both disappear at $d_{p} / w_{0}=1.00$, appear again at $d_{p} / w_{0}=1.17$ and become more pronounced at $d_{p} / w_{0}=1.33$.

At a fluence of $6.0 \mathrm{~J} / \mathrm{cm}^{2}$ (middle column) the surface of the ablated pocket appears rougher at $d_{p} / w_{0}=0.50$ and $d_{p} / w_{0}=0.67$ than at $d_{p} / w_{0}=0.83$. Furrows and ridges can be observed at $d_{p} / w_{0}=1.00$. Both disappear at $d_{p} / w_{0}=1.17$ and appear again at $d_{p} / w_{0}=1.33$.

At a fluence of $10.8 \mathrm{~J} / \mathrm{cm}^{2}$ (right column) the surface of the ablated pocket appears rougher between $d_{p} / w_{0}=0.50$ and $d_{p} / w_{0}=0.83$ than at $d_{p} / w_{0}=1.00$. Furrows and ridges can be observed at $d_{p} / w_{0}=1.17$. Both disappear at $d_{p} / w_{0}=1.33$.

The distance of the furrows agrees with the line distance $d_{l}$. 


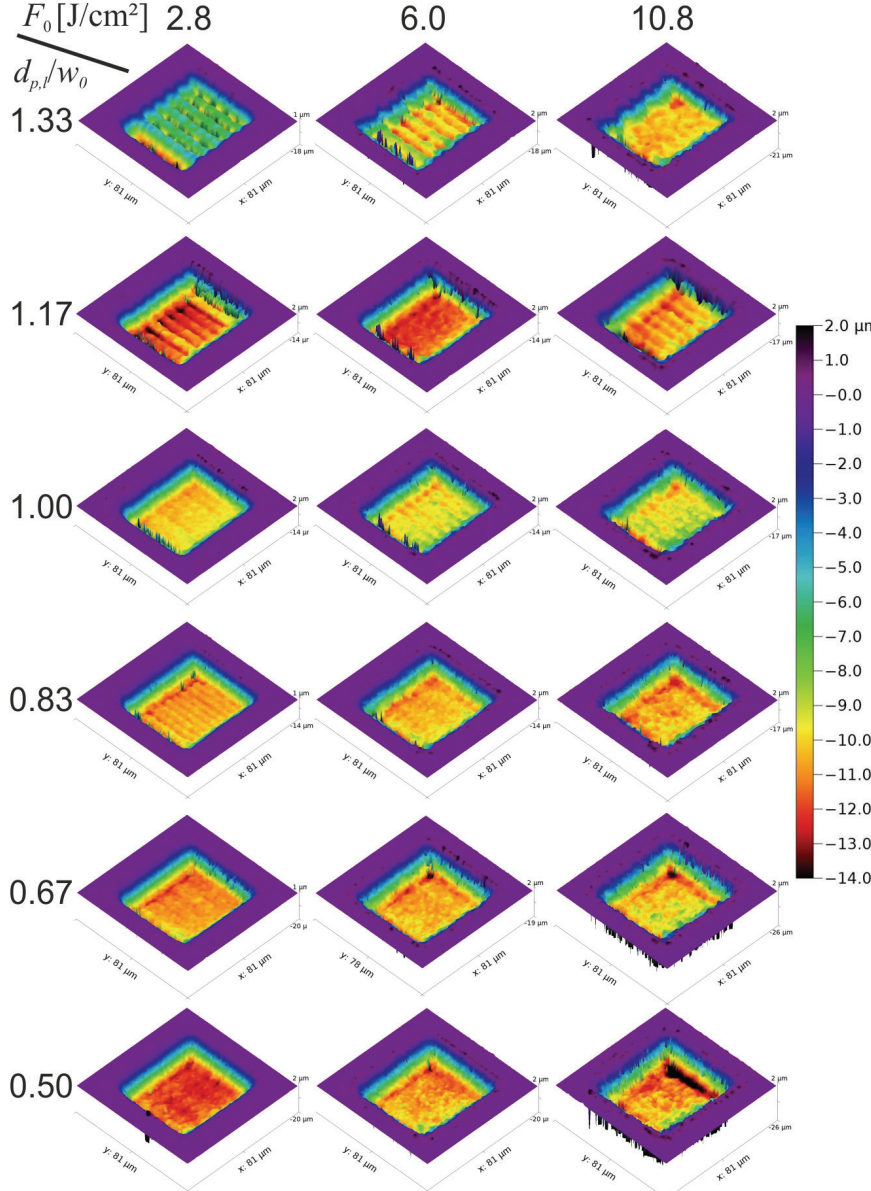

Fig. 4 Confocal microscopy images of ablated surfaces in silicon using different fluences (columns) and different ratios between pulse distances and spot radius $d_{\mathrm{p}, l} / w_{0}$ (rows). The width of the ablated squares is $50 \mu \mathrm{m}$, the depth is $10 \mu \mathrm{m}$.

The surface profiles show similar behavior for each of the three columns when increasing the pulse distance. First, the surface roughness decreases. Then, ridges and furrows appear, vanish, and appear again. Finally, their distance increases, while the furrows become deeper and the ridges higher. These observations indicate that for a fixed fluence two pulse distances exist, with which furrows and ridges can be suppressed. These two pulse distances increase with the fluence, which can be explained by the fact that higher fluences cause larger craters.

\subsection{Surface roughness of the ablated pockets}

Fig. 5 shows a contour plot of the arithmetic average surface roughness $R_{a}$ measured at the bottom of the ablated squares as a function of the ratio between pulse distance $d_{p, l}$ and focus radius $w_{0}$ and fluence. The combination where the minimum surface roughness can be achieved was determined to be at $d_{p} / w_{0}=0.67$ and at a fluence of $F_{0}=2.8 \mathrm{~J} / \mathrm{cm}^{2}$. A surface roughness of about $220 \mathrm{~nm}$ was achieved. At fluences above $8 \mathrm{~J} / \mathrm{cm}^{2}$, two surface roughness minima can be clearly identified at about $d_{p} / w_{0} \approx 0.83$ and $d_{p} / w_{0} \approx 1.33$. Only one minimum can be found at a fluence of $2.8 \mathrm{~J} / \mathrm{cm}^{2}$.

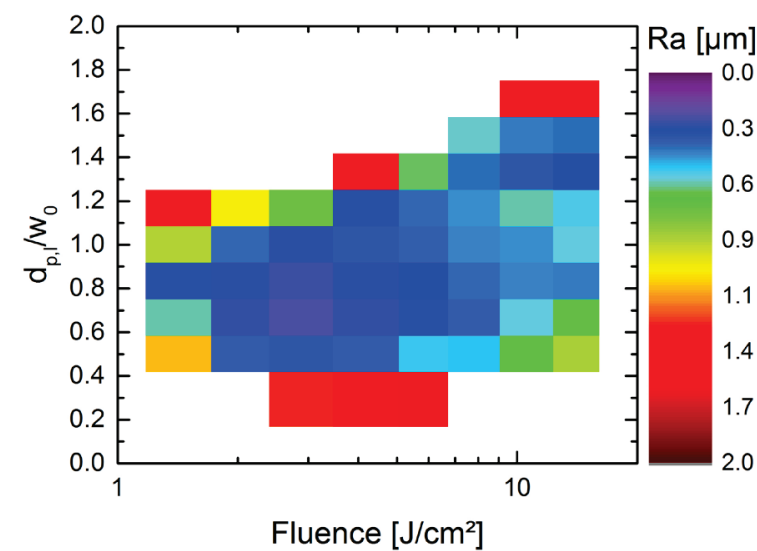

Fig. 5 Contour plot of the surface roughness $R_{a}$ at the bottom of the $10 \mu \mathrm{m}$ deep pockets in silicon as a function of the pulse distances $d_{p, l}$ in terms of the focus radius $w_{0}$ and the fluence.

It was already found that the formation of laser-induced periodic surface structures (LIPSS) increases the surface roughness at fluences below $2.8 \mathrm{~J} / \mathrm{cm}^{2}$ [3]. With increasing fluence, the amount and size of melt ejections and droplets increases, which causes the surface roughness to increase [3].

The occurrence of two surface roughness minima at about $d_{p} / w_{0} \approx 0.83$ and $d_{p} / w_{0} \approx 1.33$ for fluences above $8 \mathrm{~J} / \mathrm{cm}^{2}$ can be explained by the following observation. Fig. 4 (right column) shows the surface profile at a fluence of $10.8 \mathrm{~J} / \mathrm{cm}^{2}$ for different pulse distances. Here, furrows can be clearly observed at $d_{p} / w_{0}=1.17$, but not at $d_{p} / w_{0}=0.83$ and $d_{p} / w_{0}=1.33$. This comparison indicates that the generation of furrows increases the surface roughness. They can be avoided by either increasing or decreasing the pulse-topulse distance. At a fluences of $2.8 \mathrm{~J} / \mathrm{cm}^{2}$ the influence of the furrows on the surface roughness seems however not to be so pronounced. Only one minimum can be found.

\subsection{Optimal pulse distance as a function of fluence}

The surface roughness is plotted as a function of the ratio between pulse and line distance $d_{p, l}$ and spot radius $w_{0}$ for three different fluences in Fig. 6. The diagram shows that the minimum surface roughness can be found at a pulse distance ratio $d_{p, l} / w_{0}$ between 0.5 and 1.0 for each fluence. A parabola was fitted to data points in Fig. 6 (solid lines). The vertex of the fitted parabola indicates the optimal pulse distance ratio to minimize the surface roughness.

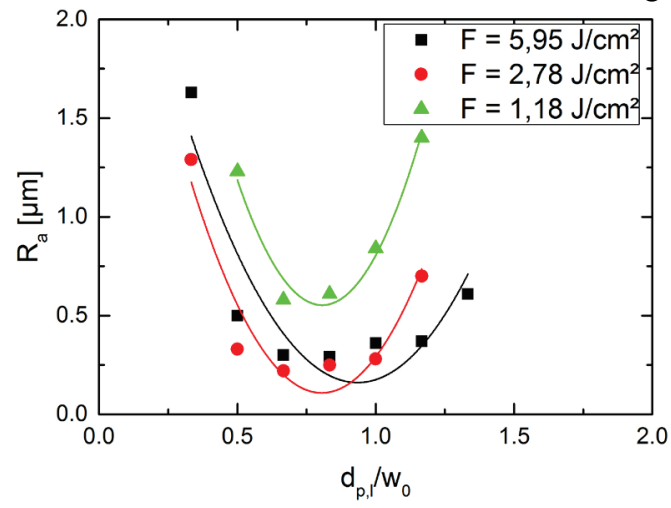

Fig. 6 Surface roughness $R_{a}$ versus $d_{p, l} / w_{0}$ for different fluences. A parabola was fitted to the data points (solid lines). 
The $\mathrm{x}$-values of the vertices of the fitted parabolas in Fig. 6 are plotted as function of the fluence in Fig. 7. Within the measured parameter range, the optimal pulse distance is $d_{p} / w_{0} \approx 0.8$ for a fluence of about $1.0 \mathrm{~J} / \mathrm{cm}^{2}$ and increases to about $d_{p} / w_{0} \approx 1.0$ for a fluence of about $14 \mathrm{~J} / \mathrm{cm}^{2}$. The trend of the data point suggests that the optimal pulse distance increases slightly with fluence. It should be taken into account that the surface roughness has two minima at fluences above $8 \mathrm{~J} / \mathrm{cm}^{2}$. Here, the fitted values represent the pulse distance between both minima.

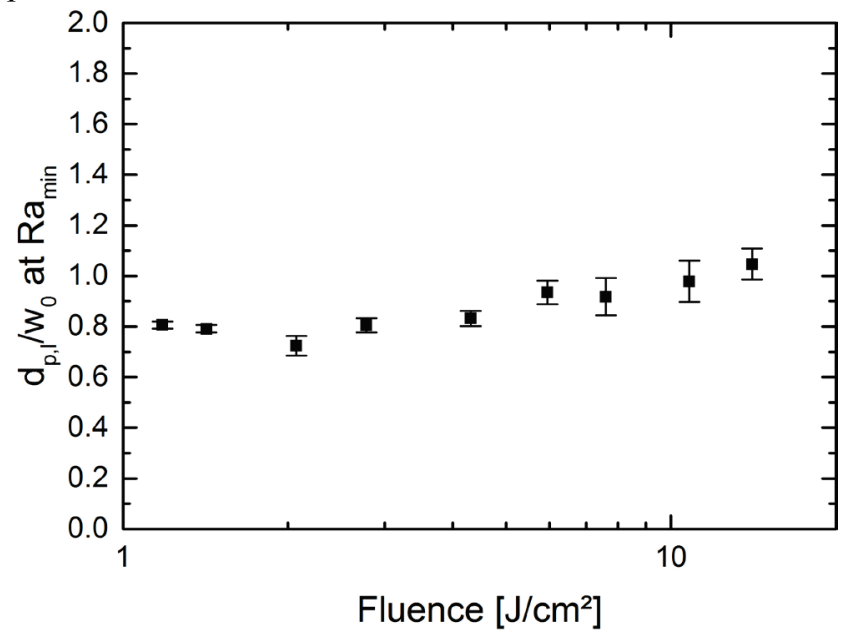

Fig. $7 \mathrm{X}$-values of the vertices of the fitted parabolas in Fig. 6 indicating the optimal pulse distance to achieve minimum surface roughness as a function of the fluence.

\section{Conclusion}

In this study, the optimal combination of pulse-to-pulse distance and fluence was determined in order to minimize the surface roughness for the ablation of Si. A Si wafer with a thickness of $525 \mu \mathrm{m}$ was irradiated using an ultrafast laser with a pulse duration of $380 \mathrm{fs}$ at a wavelength of $520 \mathrm{~nm}$ and at a pulse frequency of $200 \mathrm{kHz}$. An area of $50 \times 50 \mu \mathrm{m}^{2}$ was ablated to a depth of about $10 \mu \mathrm{m}$ by scanning the beam several times over the sample using different pulse distances $d_{p}$ and peak fluences $F_{0}$.

The maximum specific ablation rate was achieved at fluence of about $2 \mathrm{~J} / \mathrm{cm}^{2}$. The specific ablation rate then remains approximately constant with fluence.

The pulse-to-pulse distance was varied and the surface profiles were investigated. For a fixed fluence, two pulse distances can be found where the formation of furrows can be suppressed.

At a fluence of $2.8 \mathrm{~J} / \mathrm{cm}^{2}$, the global minimum of the surface roughness was determined to be about $220 \mathrm{~nm}$ at $d_{p, l}=0.67 w_{0}$. The influence of the furrows on the surface roughness seems be negligible at this fluence.

At fluences above $8 \mathrm{~J} / \mathrm{cm}^{2}$, two surface roughness minima can be clearly identified at about $d_{p, l} \approx 0.8 w_{0}$ and $d_{p, l} \approx 1.3 w_{0}$. The roughness can be decreased by avoiding the generation of furrows.

A parabola was fitted to surface roughness as function of the pulse distance for each fluence. The vertex of the fitted parabola indicates the optimal pulse distance ratio to minimize the surface roughness. The results suggest that the optimal pulse distance increases with fluence.

\section{Acknowledgment}

Thanks to all colleagues at the research center for microtechnology at the Vorarlberg University of Applied Sciences for the technical support and the interesting discussions. Thanks to Victor Matylitsky and Frank Hendricks from Spectra-Physics Austria for the close collaboration and the interesting discussions. Spectra Physics is acknowledged for the close collaboration and the financial support of the Josef Ressel Center for material processing with ultrashort pulsed lasers. The financial support by the Austrian Federal Ministry of Economy, Family and Youth and the National Foundation for Research, Technology and Development is gratefully acknowledged.

\section{References}

[1] B. Neuenschwander, G. F. Bucher, G. Hennig, C. Nussbaum, B. Joss, M. Muralt, S. Zehnder, U. W. Hunziker, and P. Schütz: International Congress on Applications of Lasers \& Electro Optics (ICALEO), (2010) p.707.

[2] B. Neuenschwander, T. Kramer, B. Lauer, and B. Jaeggi: Proc. SPIE, 93500U, (2015) p.93500U.

[3] M. Domke: Proc. SPIE, 935511, (2015) p.1.

[4] L. A. Dobrzański and A. Drygała: Journal of Materials Processing Technology, 1911-3, (2007) p.228.

[5] N. Mansour, K. Jamshidi-Ghaleh, and D. Ashkenasi: Journal of Laser Micro/Nanoengineering, 11, (2006) p.12.

[6] T.-H. Her, R. J. Finlay, C. Wu, S. Deliwala, and E. Mazur: Appl. Phys. Lett., 7312, (1998) p.1673.

[7] B. Lauer, B. Jäggi, and B. Neuenschwander: Physics Procedia, 56, (2014) p.963.

[8] V. Zorba, L. Persano, D. Pisignano, A. Athanassiou, E. Stratakis, R. Cingolani, P. Tzanetakis, and C. Fotakis: Nanotechnology, 1713, (2006) p.3234.

[9] J. M. Liu: Opt. Lett., 75, (1982) p.196.

[10] N. Bärsch, K. Körber, A. Ostendorf, and K. H. Tönshoff: Appl. Phys. A, 772, (2003) p.237.

(Received: May 20, 2015, Accepted: January 26, 2016) 